\title{
Koster-Slater model for the interface-state problem
}

\author{
M. Di Ventra, ${ }^{*}$ C. Berthod, and N. Binggeli \\ Institut de Physique Appliquée, Ecole Polytechnique Fédérale de Lausanne, CH-1015 Lausanne, Switzerland
}

(Received 1 May 2000)

\begin{abstract}
A Koster-Slater approach to the problem of localized states at semiconductor interfaces has been developed. It allows us to relate the existence and/or the energy position of interface states to some essential bulk features of the constituent materials and some interface-bonding parameters. The condition for the existence of localized states and the relevance of the model will be discussed comparing the predictions entailed by the latter with the results of $a b$ initio calculations on the Ge/GaAs (110) interface.
\end{abstract}

The study of interface states of a given heterostructure has long been recognized as a key element for the improvement of optoelectronic devices. Depending on their specific nature, i.e., symmetry and binding energy, these states can act as recombination centers and contribute to the degradation of device performance. Theoretical investigation of heterojunction interface states began with the pioneering work of Baraff $e$ t $a l .{ }^{1}$ on the (100) GaAs/Ge interface. Since then, several different interfaces have been studied, with special emphasis on the interface-state problem. ${ }^{1-4}$ Different key aspects of this problem, namely the importance of the local interfacial atomic structure and that of the band structures of the bulk constituents in determining the interface-state spectrum, have been highlighted by full-fledged atomic-scale computations on one hand and Green's function formulations on the other hand. $^{2-5}$ To our knowledge, however, a complete understanding of the origin of interface states and the conditions of their existence is still missing.

The available calculations have shown that for a given interface, intrinsic localized states are generally found only along specific lines and/or at specific points of the Brillouin zone (BZ). ${ }^{1-4}$ They may appear above or below the continuous energy spectrum of a given set of projected bands of the two bulk semiconductors depending on the strength and sign of some short-range potentials at the interface. ${ }^{2,3}$ On the other hand, the physical properties of a given interface state must depend on the electronic structure of the bulk constituents. In fact, by analogy with the problem of deep defect levels in a bulk semiconductor, ${ }^{6}$ the existence of interface states should strongly depend on the dispersion or density of states (DOS) of the electronic bands of the two bulk materials. ${ }^{4,5}$ Finally, another important parameter which is also expected to affect the energy position of interface states is the energy band lineup between the two bulk solids. Predicting the existence and the position of interface states from the above quantities could help designing better devices and provide a deeper understanding of the mechanisms of interface-state formation.

In this paper we derive a simple analytic criterion for the existence of interface states, based on which localized states and resonances can be predicted from the above quantities. Our starting point relies on the physical assumption that an interface can be regarded as a perturbation of the bonding structures of two different bulk materials. ${ }^{4}$ Also, since the perturbation involved is short range, we may formulate the problem in terms of few perturbed layers and derive a model similar to the one Koster and Slater introduced for point defects in semiconductors. ${ }^{6}$ We plot in Fig. 1 a scheme of the interface between two semiconductors (lattice matched) $A$ and $B .^{7}$ We construct the interface as a combination of two semi-infinite $A$ and $B$ crystals, and assume that only the "interface layers" of each semiconductor (indicated as $\mathbf{R}_{0}^{A}$ and $\mathbf{R}_{0}^{B}$ in Fig. 1) are perturbed by the interface formation. Moreover, to simplify as much as possible the equations, we refer in the following to one band $\varepsilon_{A(B)}(\mathbf{k})$ for each bulk semiconductor. The generalization to the multiband case is straightforward, and will be discussed later. The interface Hamiltonian then reads (in atomic units):

$$
H=-\frac{\nabla^{2}}{2}+\sum_{\alpha}\left[U_{\alpha}(\mathbf{r})+\Delta u_{\alpha}^{0}(\mathbf{r})\right]+\left[\theta(z)-\frac{1}{2}\right] \Delta V
$$

where $\alpha=A, B$. The terms $U_{\alpha}$ are the crystal potentials of the two semiconductors in the half space only, corresponding to the semi-infinite bulk regions of the interface, and can be expressed as a sum of atomic-layer potentials $u_{\alpha}\left(\mathbf{R}_{j}^{\alpha}, \mathbf{r}\right)$ in each bulk region. The terms $\Delta u_{\alpha}^{0}(\mathbf{r})$ are the perturbations to the bulk potentials induced by the junction at the interface sites $\mathbf{R}_{0}^{\alpha}$ (see Fig. 1). These terms take into account the charge transfer at the interface, i.e., the variations of the interface potential with respect to a superposition of bulk crystal potentials. $\theta(z)$ is the step function which indicates the change in the electrostatic potential $\Delta V$ at the interface. The

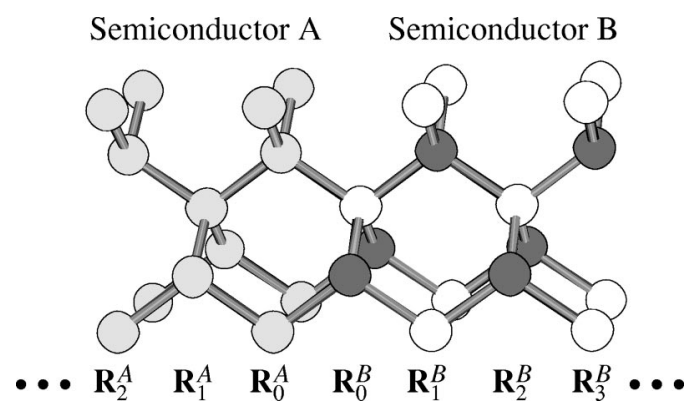

FIG. 1. Schematic plot of the interface between a semiconductor $A$ and a semiconductor $B$ (in this case the Ge/GaAs (110) interface). $\mathbf{R}_{i}^{\alpha}$ indicate the positions of the atomic layers in each material. 
potentials $U_{\alpha}, u_{\alpha}$, and $\Delta u_{\alpha}$ are measured with respect to the average electrostatic potential in the corresponding crystal $\alpha$.

The Bloch functions of each bulk material $\psi_{\mathbf{k}}^{\alpha}(\mathbf{r})$ satisfy the equation $H_{\alpha} \psi_{\mathbf{k}}^{\alpha}(\mathbf{r})=\varepsilon_{\alpha}(\mathbf{k}) \psi_{\mathbf{k}}^{\alpha}(\mathbf{r})$, with

$$
H_{\alpha}=-\frac{\nabla^{2}}{2}+U_{\alpha}(\mathbf{r})+\sum_{j=0}^{\infty} u_{\alpha}\left(-\mathbf{R}_{j}^{\alpha}, \mathbf{r}\right)
$$

The wave vectors $\mathbf{k}$ may be written as $\mathbf{k}=\left(\overline{\mathbf{k}}, k_{z}\right)$, where $\overline{\mathbf{k}}$ belongs to the two-dimensional BZ (2DBZ) of the interface and $k_{z}$ is along the heterojunction growth direction. We assume standard periodic boundary conditions over $N$ layers for the bulk semiconductors $A$ and $B$, so that $k_{z}=k_{i}$, with $i$ $=0, \ldots, N-1$.

From the Bloch functions of each bulk we can build a set of isolated Wannier functions centered on each layer and localized over a distance of the order of the lattice spacing $d$ between two nearest-neighbor planes: ${ }^{8}$

$$
w_{j}^{\alpha}(\overline{\mathbf{k}}, \mathbf{r})=\frac{1}{\sqrt{N}} \sum_{k_{z}} e^{-i \mathbf{k} \mathbf{R}_{j}^{\alpha}} \psi_{\mathbf{k}}^{\alpha}(\mathbf{r}) \theta\left(\frac{N d}{2}-\left|z-z_{j}^{\alpha}\right|\right),
$$

where $\mathbf{R}_{j}^{\alpha}=\left(0,0, z_{j}^{\alpha}\right)$ indicate the positions of the atomic layers in the two bulk regions. We then choose as a basis for interface states with a given $\overline{\mathbf{k}}$ the set

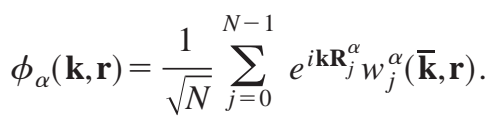

A general interface state can thus be written as

$$
\Psi(\overline{\mathbf{k}}, \mathbf{r})=\sum_{k_{z}} c_{A}(\mathbf{k}) \phi_{A}(\mathbf{k}, \mathbf{r})+\sum_{k_{z}} c_{B}(\mathbf{k}) \phi_{B}(\mathbf{k}, \mathbf{r})
$$

The wave-vector $\overline{\mathbf{k}}$ is a good quantum number, since the system retains its periodicity in planes parallel to the interface. In the following, we thus drop it from the notation, and a given $\overline{\mathbf{k}}$ will be understood. The $k_{z}$ and $j$ summations in Eqs. (3)-(5) refer to the $k_{z}$ values and the layer positions of each bulk only, and we assume that the Wannier functions of bulks $A$ and $B$ are orthogonal, i.e., $\left\langle w_{i}^{A} \mid w_{j}^{B}\right\rangle=0$. Orthogonality of the Wannier functions of the two different materials implies:

$$
\left\langle\phi_{\alpha}\left(k_{z}\right) \mid \phi_{\beta}\left(k_{z}^{\prime}\right)\right\rangle=\delta_{k_{z} k_{z}^{\prime}} \delta_{\alpha \beta}
$$

The solution of the interface problem $H \Psi=E \Psi$ then reduces to the evaluation of the sets of equations

$$
\left\langle\phi_{\alpha}\left(k_{z}\right)|H-E| \Psi\right\rangle=0
$$

for $\alpha=A, B$. Taking into account the orthogonality condition (6) and including up to second-nearest-neighbor interactions and two-center integrals ${ }^{9}$ in the evaluation of the matrix elements of $H$ onto the basis set (3), it is straightforward to show that

$$
\begin{gathered}
\left\langle\phi_{A}\left(k_{z}\right)|H-E| \phi_{A}\left(k_{z}^{\prime}\right)\right\rangle \\
=\left[\varepsilon_{A}(\mathbf{k})-E-\Delta V / 2\right] \delta_{k_{z} k_{z}^{\prime}}+\frac{V_{A}}{N} e^{-i\left(k_{z}-k_{z}^{\prime}\right) z_{0}^{A}} \\
-\frac{\Delta \varepsilon_{A}}{N}\left[e^{i\left(k_{z}+k_{z}^{\prime}\right) z_{0}^{A}}+e^{-i\left(k_{z}+k_{z}^{\prime}\right) z_{0}^{A}}\right],
\end{gathered}
$$

where

$$
V_{A}=\left\langle w_{0}^{A}\left|u_{B}\left(-\mathbf{R}_{0}^{A}\right)-u_{A}\left(-\mathbf{R}_{0}^{A}\right)+\Delta u_{A}^{0}\right| w_{0}^{A}\right\rangle
$$

and

$$
\Delta \varepsilon_{A}=\left\langle w_{0}^{A}\left|H_{A}\right| w_{1}^{A}\right\rangle .
$$

The equivalent terms for the $B$ states can be obtained from Eqs. (8) $-(10)$ by interchanging $A$ with $B$ and $-\Delta V / 2$ with $+\Delta V / 2$. The first term on the right-hand side of Eq. (8) is diagonal, and contains the band energy $\varepsilon_{\alpha}(\mathbf{k})$ measured with respect to the average electrostatic potential in bulk $\alpha$. The second term expresses the expectation value of the difference of interface-layer potentials $u_{B}\left(-\mathbf{R}_{0}^{A}, \mathbf{r}\right)$ and $u_{A}\left(-\mathbf{R}_{0}^{A}, \mathbf{r}\right)$ of the two materials over Wannier functions located on only one side of the interface. The last term contains the matrix element of the Hamiltonian of the bulk crystal $H_{\alpha}$ between Wannier functions located on neighboring sites.

The interbulk matrix elements read:

$$
\left\langle\phi_{A}\left(k_{z}\right)|H-E| \phi_{B}\left(k_{z}^{\prime}\right)\right\rangle=\frac{1}{N}\left(V_{A B}+\Delta \varepsilon_{A B}\right) e^{-i\left(k_{z}-k_{z}^{\prime}\right) z_{0}^{A},}
$$

where

$$
\begin{aligned}
V_{A B}= & \frac{1}{2}\left\langle w_{0}^{A}\left|u_{A}\left(+\mathbf{R}_{0}^{A}\right)-u_{B}\left(+\mathbf{R}_{0}^{A}\right)\right| w_{0}^{B}\right\rangle \\
& +\frac{1}{2}\left\langle w_{0}^{A}\left|u_{B}\left(-\mathbf{R}_{0}^{A}\right)-u_{A}\left(-\mathbf{R}_{0}^{A}\right)\right| w_{0}^{B}\right\rangle
\end{aligned}
$$

and

$$
\Delta \varepsilon_{A B}=\left\langle w_{0}^{A}\left|\frac{1}{2}\left(H_{A}+H_{B}\right)\right| w_{0}^{B}\right\rangle .
$$

These terms couple the two interface layers in the heterojunction. The same notation as for the intrabulk terms has been used, making clear the meaning of each term in Eqs. (11)-(13).

Multiplying Eq. (7) by $e^{i k_{z} z_{0}^{\alpha}} /\left[E-\varepsilon_{\alpha}(\mathbf{k}) \mp \Delta V / 2\right]$ and summing over $k_{z}$, we finally obtain the following condition for the existence of localized states:

$$
\begin{gathered}
\left(1-V_{A} G_{0}^{A}+\Delta \varepsilon_{A} S_{0}^{A}\right)\left(1-V_{B} G_{0}^{B}+\Delta \varepsilon_{B} S_{0}^{B}\right) \\
-\left(V_{A B}+\Delta \varepsilon_{A B}\right)^{2} G_{0}^{A} G_{0}^{B}=0
\end{gathered}
$$

where

$$
\begin{aligned}
G_{0}^{A}(\overline{\mathbf{k}}, E) & =\frac{1}{N} \sum_{k_{z}} \frac{1}{E-\varepsilon_{A}(\mathbf{k})-\Delta V / 2} \\
& =\int \frac{D_{0}^{A}(\overline{\mathbf{k}}, \varepsilon) d \varepsilon}{E-\varepsilon-\Delta V / 2}
\end{aligned}
$$


and

$$
S_{0}^{A}(\overline{\mathbf{k}}, E)=\frac{1}{N} \sum_{k_{z}} \frac{e^{i k_{z} d}}{E-\varepsilon_{A}(\mathbf{k})-\Delta V / 2},
$$

and $G_{0}^{B}$ and $S_{0}^{B}$ are obtained by replacing $A$ with $B$ and $-\Delta V / 2$ with $+\Delta V / 2$ in Eqs. (15) and (16). $G_{0}^{\alpha}$ is the usual (retarded) Green's function of bulk $\alpha,{ }^{10}$ and $D_{0}^{\alpha}$ the corresponding DOS per unit cell integrated along $k_{z}$. We note that in the above expressions $\Delta V$ may be replaced, e.g., by the $A / B$ valence-band offset when the band energies $\varepsilon_{A(B)}(\mathbf{k})$ are measured relative to their respective $A(B)$ valence-band edge and $E$ is measured with respect to the average between the $A$ and $B$ valence-band edges in the heterojunction.

Equation (14) can be further simplified if only linear terms in the expansion of the bulk bands are retained, i.e., the terms $\Delta \varepsilon_{\alpha} S_{0}^{\alpha} \propto O\left(\Delta \varepsilon_{\alpha}^{2}\right)$ and $\Delta \varepsilon_{A B}^{2}$ are neglected. The equation for the existence of interface states then reads:

$$
1-V_{A} G_{0}^{A}-V_{B} G_{0}^{B}-\left[\left(V_{A B}+2 \Delta \varepsilon_{A B}\right) V_{A B}-V_{A} V_{B}\right] G_{0}^{A} G_{0}^{B}=0 .
$$

The energy $E$ is imaginary inside the bands and real otherwise. It is straightforward to generalize the condition for the existence of interface states to the case of many bands for each semiconductor. ${ }^{11}$ In this case a more complex matrix equation states the multiband interactions which govern the existence of interface states. We note, however, that in the special cases that will be addressed below, where the matrix $V_{A B} \approx 0$ (nearly isolated $s$-band) or when $V_{A} \approx 0, V_{B} \approx 0$ and $G_{0}^{A}, G_{0}^{B}$ are multiples of the unity matrix ( $p$-valence bands of cubic semiconductor at the $\overline{\mathrm{X}}, \overline{\mathrm{M}}$, and $\overline{\mathrm{X}}^{\prime} 2 \mathrm{DBZ}$ points of the (110) interface), the general matrix equation is equivalent to a set of one-dimensional equations of the form (17). We will therefore concentrate on Eq. (17) in what follows.

Condition (17) reduces to the usual Koster-Slater relation ${ }^{6}$ $1-V G_{0}=0$ for point defects when only one semiconductor and on-site interactions are retained, $V$ being the potential induced by the point-defect perturbation in the host described by $G_{0}$. Given the linear relationship between the oneparticle Green's function and the DOS, we find from Eq. (17) that, for a given set of parameters $\left(V_{A}, V_{B}, V_{A B}^{2}\right.$ $+2 \Delta \varepsilon_{A B} V_{A B}$ and $\left.\Delta V\right)$, the existence of bound states and resonances depends essentially on: (i) the strength of the DOS of each band with respect to the corresponding on-site parameter $V_{A(B)}^{-1}$, and (ii) the amplitude of the product of the DOS's of the two bulk bands with respect to the interface coupling parameter $\left(V_{A B}^{2}+2 \Delta \varepsilon_{A B} V_{A B}\right)^{-1}$. Interface states may be pushed above and/or below the bulk bands depending not only on the strength of the on-site terms $V_{A}$ and $V_{B}$ at the interface, but also on the relative strength of these terms and the potential term $V_{A B}$ due to the different chemical bonding at the interface. In fact, when $V_{A}=V_{B}=0, V_{A B}$ will push states both above and below the bulk bands. It should be noted, however, that when the band offset is not vanishing, even in a one-dimensional model, the on-site perturbations induced by the interface may induce resonances rather than bound states. This is in contrast to the case of point defects, or isovalent layer impurities, where a state is always bound in one dimension. ${ }^{12}$

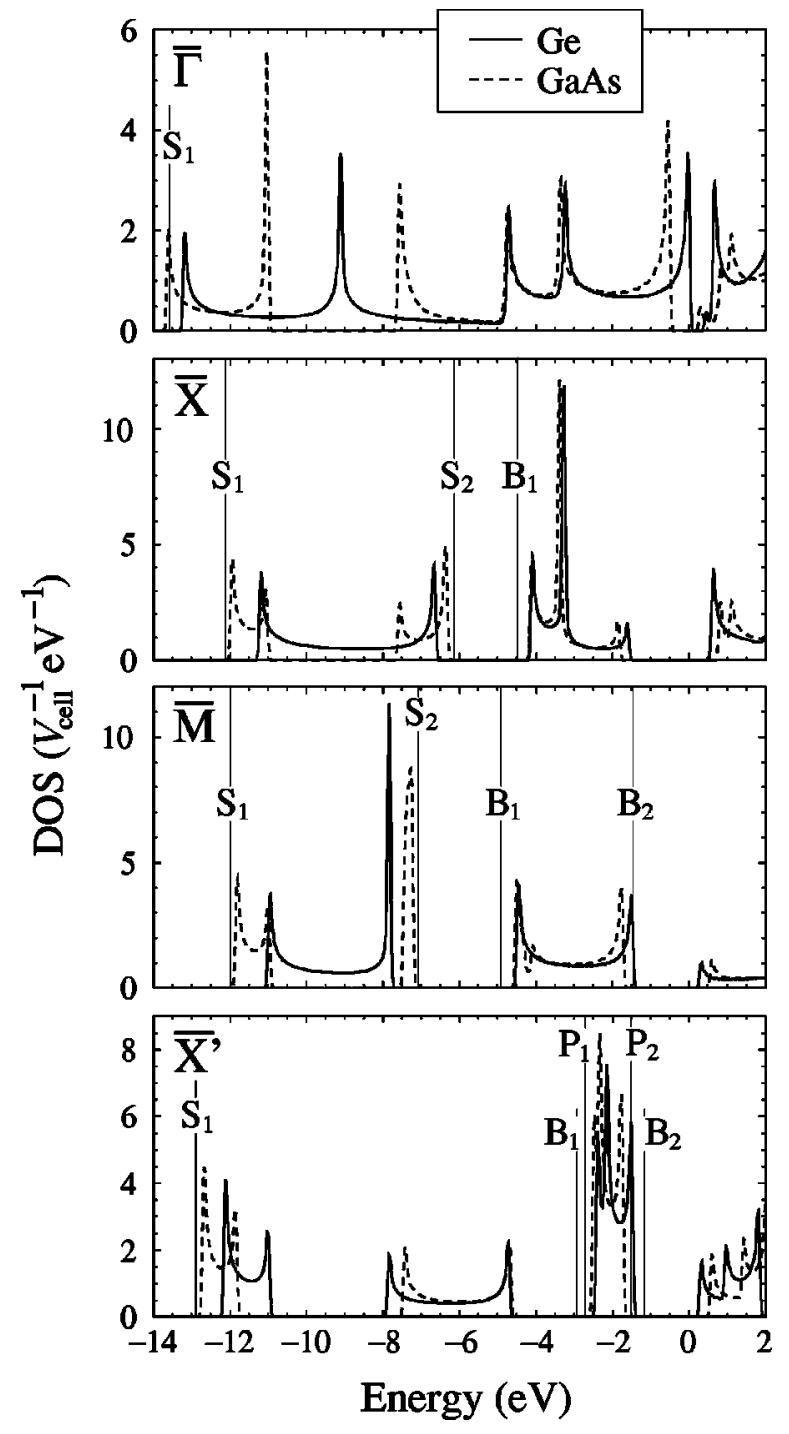

FIG. 2. Wave-vector-resolved density of states (DOS) of bulk $\mathrm{Ge}$ and GaAs for the four high-symmetry points of the (110) 2DBZ, and integrated along a line in $k$-space parallel to the [110] crystallographic direction. The two DOS are aligned using the calculated valence-band offset of $0.52 \mathrm{eV}$ at the (110) Ge/GaAs interface. The volume of normalization is the GaAs unit-cell volume. The vertical lines indicate the energy position of the interface states, and the notation is the same as in Ref. 2. The degeneracies of the bulk Ge (GaAs) valence-band DOS features, given in order of increasing energy, are: $2,2(1,1,2)$ at $\overline{\mathrm{X}}$ and $\overline{\mathrm{M}}$, and $1,1,2(1,1,2)$ at $\overline{\mathrm{X}}^{\prime}$.

With the above considerations in mind, we can now identify the regions and points of the 2DBZ, for a given interface, where interface states are more likely to appear. We have chosen as a prototypical example the Ge/GaAs (110) interface studied by Pickett et al. ${ }^{2}$ In particular, we refer to Fig. 1 of Ref. 2 for the notations and results on the interface states. In view of the relation between Green's function and DOS, we elected to directly investigate the DOS features of the bulk materials. We therefore looked at the valence DOS of both Ge and GaAs bulks for a given point of the (110) 2DBZ integrated along a line in $k$-space parallel to the growth direction. The result is plotted in Fig. 2 for the four highsymmetry points of the (110) 2DBZ. We also indicated in Fig. 2 the energy position of the localized and resonant states 
we found for the specific interface. The bulk DOS's have been calculated using tetragonal supercells of four atoms oriented along the (110) direction. For each point in the 2DBZ we sampled the (110) direction with $101 \mathrm{k}$-points distributed on a uniform grid. A Gaussian broadening of $0.1 \mathrm{eV}$ has been used for the DOS's plot. The zero of energy has been fixed at the top of the Ge valence band. ${ }^{13}$

The bottom edge of the $s$-band of GaAs is well separated from the Ge bands. Thus, the As-related $s$-interface-state $\left(\mathrm{S}_{1}\right.$ state in Ref. 2) clearly originates from the bottom-edge states of GaAs, and is induced by an on-site potential. The $V_{\mathrm{As}}$ on-site-potential component is attractive due to the attractive ionic potential of the nearest-neighbor Ge atoms, and is responsible for the existence of this state. Due to the energy separation of the GaAs and Ge band edges, and also to the $s$ symmetry of the corresponding bottom-edge bulk states, the interaction term $V_{A B}$ does not contribute significantly in Eq. (17). This is also confirmed by the spherical character of the $S_{1}$ states. Moving upwards in energy, we see that the $S_{2}$ state derives mainly from the $\overline{\mathrm{X}}$ and $\overline{\mathrm{M}}$ edges at about $-7 \mathrm{eV}$ of GaAs. The on-site matrix element $V_{\mathrm{Ga}}$ is repulsive in this energy region: the nearest $\mathrm{Ge}$ atoms produce a repulsive potential on the Wannier function at the Ga sites. For the interaction term $V_{A B}$, the same considerations as for the As $s$ states are valid. We note that at the $\bar{\Gamma}$ and $\overline{\mathrm{X}}^{\prime}$ points, no comparable DOS features are detected at similar energies.

At $\bar{X}^{\prime}$ two pronounced and strongly overlapping DOS features of $\mathrm{Ge}$ and $\mathrm{GaAs}$ are visible, with band edges at about $-2.5 \mathrm{eV}$ and $-1.5 \mathrm{eV}$. The lower edges contribute to the $\mathrm{B}_{1}$ and $\mathrm{P}_{1}$ states, and the upper ones to the $\mathrm{P}_{2}$ and $\mathrm{B}_{2}$ states. However, only the $\mathrm{B}_{1}$ and $\mathrm{B}_{2}$ states can be followed along the 2DBZ up to the $\overline{\mathrm{X}}$ point. The strong overlap near the band edges and the $p$ character of the wave functions at these edges make the contribution of the $V_{A B}$ term important, as evidenced by the mixed Ge-GaAs character of the resulting interface states. These states are localized by the attractive ionic potential in the $\mathrm{Ge}-\mathrm{As}$ bonding region and the repulsive potential in the $\mathrm{Ge}-\mathrm{Ga}$ bonding region. At the $\bar{\Gamma}$ point no bound state is present in the fundamental gap. This is due to the attractive character of the on-site matrix elements $V_{\mathrm{As}}$ and $V_{\mathrm{Ge}}$ associated with the As- $p$ and Ge- $p$ Wannier functions of the top of the GaAs and Ge valence bands, and to the finite value of the valence-band offset. The coupling term $V_{A B}$ has thus not enough strength to push a state in the gap. Similar considerations apply to other interfaces studied in the literature, ${ }^{2-4}$ thus giving us confidence in the validity of the approach. We stress that the different interface-bonding parameters, defined by Eqs. (9), (12), and (13), can be obtained from relatively simple models, such as tight-binding approaches. ${ }^{4}$ With the above parameters, the existence of localized states at a given interface can then be inferred from Eq. (17) without the need to perform a fullfledged interface-state calculation.

We finally note that if some intermixing is present at the interface and involves only the first layer of atoms of each semiconductor, Eq. (17) is still valid if the different parameters are evaluated as weighted averages of those of the two semiconductors. If the intermixing extends over the second layer of the interface (say, e.g., diffusion of atoms $A$ in material $B$ ) then the effect of material $A$ on the states localized in semiconductor $B$ may be neglected to first order, and Eq. (17) reduces to the usual Koster-Slater relation. Indeed, the local density of states of the bulk is generally recovered after 2-3 layers from the interface, and the formation of interface states, to a good approximation, may be inferred from the analysis of impurity states in the corresponding bulk.
*Present address: Department of Physics, Virginia Polytechnic Institute and State University, Blacksburg, Virginia, 24061.

${ }^{1}$ G. A. Baraff, J. A. Appelbaum, and D. R. Hamann, Phys. Rev. Lett. 38, 237 (1977); J. Vac. Sci. Technol. 14, 999 (1977).

${ }^{2}$ W. E. Pickett, S. G. Louie, and M. L. Cohen, Phys. Rev. Lett. 39, 109 (1977).

${ }^{3}$ W. E. Pickett and M. L. Cohen, Phys. Rev. B 18, 939 (1978); W. E. Pickett, S. G. Louie, and M. L. Cohen, Phys. Rev. B 17, 815 (1978)

${ }^{4}$ J. Pollmann and S. T. Pantelides, Phys. Rev. B 18, 5524 (1978); ibid. 21, 709 (1980); Solid State Commun. 30, 621 (1979).

${ }^{5}$ V. R. Velasco and F. Garcia-Moliner, Phys. Scr. 34, 256 (1986); A.-B. Chen, Y.-M. Lai-Hsu, and W. Chen, Phys. Rev. B 39, 923 (1989); H. L. Skriver and N. M. Rosengaard, ibid. 43, 9538 (1991).

${ }^{6}$ G. F. Koster and J. C. Slater, Phys. Rev. 96, 1208 (1954).

${ }^{7}$ The existence criterion we derive for interface states [Eq. (17)] is independent of the lattice-matched condition.

${ }^{8}$ The $\theta$ function is introduced to suppress the periodic images of the Wannier function at $z_{j} \pm n N d, n=1,2, \ldots$.
${ }^{9}$ For the potential variations $\Delta u$ and $\Delta V$, only on-site interactions are considered.

${ }^{10}$ E. N. Economou, Green's Functions in Quantum Physics (Springer, Berlin, 1979).

${ }^{11}$ For the multiband case, the condition for the existence of interface states obtained neglecting terms of order $O\left(\Delta \varepsilon_{\alpha}^{2}\right)$ is:

$$
\left|\begin{array}{lr}
\mathbf{1}-G_{0}^{A} V_{A} & -G_{0}^{A}\left(V_{A B}+\Delta \varepsilon_{A B}\right) \\
-G_{0}^{B}\left(V_{A B}+\Delta \varepsilon_{A B}\right)^{T} & \mathbf{1}-G_{0}^{B} V_{B}
\end{array}\right|=0,
$$

where $V_{\alpha}, V_{A B}, \Delta \varepsilon_{A B}$, and $G_{0}^{\alpha}$ are the matrices whose elements are obtained from Eqs. (9), (12), (13), and (15), respectively, by introducing the band indexes $\left(n, n^{\prime}\right)$ of the Wannier functions, or the indexes $n$ of the energy bands (for $G_{0}^{\alpha}$, diagonal matrix).

${ }^{12} \mathrm{We}$ refer here to the case of local potentials.

${ }^{13} \mathrm{We}$ used an energy cutoff of $20 \mathrm{Ry}$, and Troullier-Martins pseudopotentials [N. Troullier and J. L. Martins, Phys. Rev. B 43, 1993 (1991)] instead of the semiempirical ones of Ref. 2. This, however, does not change the physics and the trends of the results. 